\title{
A Cesium optical atomic clock with high optical frequency stability
}

\author{
Kazunari Tokuhira $^{\text {a) }}$, Fumitaka Suzuki, Masato Yoshida, \\ and Masataka Nakazawa \\ Research Institute of Electrical Communication, Tohoku University, \\ 2-1-1 Katahira, Aoba-ku, Sendai, Miyagi 980-8577, Japan \\ a)tokuhira@riec.tohoku.ac.jp
}

Abstract: We describe the greatly improved performance of a $1.5 \mu \mathrm{m}$ optical frequency stabilized Cs atomic clock composed of a mode-hopfree mode-locked fiber laser and an acetylene optical frequency standard. The output pulse width was shortened from 7.1 to 3.4 ps by employing a phase modulator instead of an intensity modulator as a mode-locker. The optical frequency stability for an averaging time of $100 \mathrm{~s}$ was also improved from $1.8 \times 10^{-11}$ to $6.3 \times 10^{-12}$ by precisely controlling the laser cavity temperature.

Keywords: Cs optical atomic, optical frequency stabilization, short pulse

Classification: Optoelectronics, Lasers and quantum electronics, Ultrafast optics, Silicon photonics, Planar lightwave circuits

\section{References}

[1] C. Schmidt-Langhorst, R. Ludwig, D.-D. Grob, L. Molle, M. Seimetz, R. Freund, and C. Schubert, "Generation and coherent time-division demultiplexing of up to $5.1 \mathrm{~Tb} / \mathrm{s}$ single-channel 8-PSK and 16-QAM signals," National Fiber Optic Engineers Conference (NFOEC), San Diego, USA, PDPC6, March 2009.

[2] K. Kasai, T. Omiya, P. Guan, M. Yoshida, T. Hirooka, and M. Nakazawa, "Single-channel 400-Gb/s OTDM-32 RZ/QAM coherent transmission over $225 \mathrm{~km}$ using an optical phase-locked loop technique," IEEE Photon. Technol. Lett., vol. 22, no. 8, pp. 562-564, April 2010.

[3] Z. Jiang, C. Huang, D. E. Leaird, and A. M. Weiner, "Optical arbitrary waveform processing of more than 100 spectral comb lines," Nat. Photonics, vol. 1, pp. 463-467, Aug. 2007.

[4] G. G. Ycas, F. Quinlan, S. A. Diddams, S. Osterman, S. Mahadevan, S. Redman, R. Terrien, L. Ramsey, C. F. Bender, B. Botzer, and S. Sigurdsson, "Demonstration of on-sky calibration ofastronomical spectra using a $25 \mathrm{GHz}$ near-IR laser frequency comb," Opt. Express, vol. 20, no. 6, pp. 6631-6643, March 2012.

[5] S. M. Foreman, K. W. Holman, D. D. Hudson, D. J. Jones, and J. Ye, "Remote transfer of ultrastable frequency references via fiber networks," Rev. Sci. Instrum., vol. 78, 021101, Feb. 2007.

[6] D. J. Jones, S. A. Diddams, J. K. Ranka, A. Stentz, R. S. Windeler, J. L. Hall, and S. T. Cundiff, "Carrier-envelope phase control of femtosec- 
ond mode-locked lasers and direct optical frequency synthesis," Science, vol. 288, pp. 635-639, April 2000.

[7] B. R. Washburn, S. A. Diddams, N. R. Newbury, J. W. Nicholson, M. F. Yan, and C. G. Jørgensen, "Phase-locked, erbium-fiber-laser-based frequency comb in the near infrared," Opt. Lett., vol. 29, no. 3, pp. 250-252, Feb. 2004.

[8] J. Chen, J. W. Sickler, P. Fendel, E. P. Ippen, F. X. Kärtner, T. Wilken, R. Holzwarth, and T. W. Hänsch, "Generation of low-timing-jitter femtosecond pulse trains with $2 \mathrm{GHz}$ repetition rate via external repetition rate multiplication," Opt. Lett., vol. 33, no. 9, pp. 959-961, May 2008.

[9] M. Nakazawa and K. Suzuki, "Cesium optical clock: an optical pulse that tells the time," Opt. Lett., vol. 26, no. 9, pp. 635-637, May 2001.

[10] T. Hirayama, M. Yoshida, M. Nakazawa, K. Hagimoto, and T. Ikegami, "A mode-locked laser-type optical atomic clock with an optically pumped Cs gas cell," Opt. Lett., vol. 32, no. 10, pp. 1241-1243, May 2007.

[11] M. Yoshida, F. Suzuki, T. Morisaki, and M. Nakazawa, "Absolutely optical-frequency-stabilized cesium optical atomic clock," 17th Microoptics Conference, Sendai, Japan, B-2, pp. 1-2, Nov. 2011.

[12] M. Nakazawa, E. Yoshida, and Y. Kimura, "Ultrastable harmonically and regeneratively modelocked polarization-maintaining erbium fibre ring laser," Electron. Lett., vol. 30, no. 19, pp. 1603-1604, Sept. 1994.

[13] M. Yoshida, T. Yaguchi, S. Harada, and M. Nakazawa, "A 40 GHz regeneratively and harmonically mode-locked erbium-doped fiber laser and its longitudinal-mode characteristics," IEICE Trans. Electron., vol. E87-C, no. 7, pp. 1166-1172, July 2004.

[14] T. Morisaki, M. Yoshida, and M. Nakazawa, "Optical frequency-tunable Cs atomic clock with a $9.19 \mathrm{GHz}$ mode-hop-free fiber laser," IEICE Electron. Express, vol. 7, no. 21, pp. 1652-1658, Nov. 2010.

[15] Y. Ohuchi, H. Suga, T. Suzuki, M. Uchino, K. Takahei, M. Tuda, and Y. Saburi, "A high-stability laser-pumped Cs gas-cell frequency standard," 2000 IEEE/EIA Int. Freq. Control Symp. and Exhibition, Kansas City, USA, pp. 651-655, June 2000.

[16] L. L. Lewis and M. Feldman, "Optical pumping by lasers in atomic frequency standards," 35th Annu. Symp. Freq. Control, USAERADCOM, Ft. Monmouth, NJ, 07703, pp. 612-624, May 1981.

[17] M. Nakazawa and M. Yoshida, "Scheme for independently stabilizing the repetition rate and optical frequency of a laser using a regenerative mode-locking technique," Opt. Lett., vol. 33, no. 10, pp. 1059-1061, May 2008.

[18] K. Kasai, A. Suzuki, M. Yoshida, and M. Nakazawa, "Performance improvement of an acetylene $\left(\mathrm{C}_{2} \mathrm{H}_{2}\right)$ frequency-stabilized fiber laser," IEICE Electron. Express, vol. 3, no. 22, pp. 487-492, Nov. 2006.

[19] D. W. Allan, "Statistics of atomic frequency standards," Proc. IEEE, vol. 54, no. 2, pp. 221-230, Feb. 1966.

\section{Introduction}

A stable optical short pulse train (or optical comb) with a $\mathrm{GHz}$ repetition rate at $1550 \mathrm{~nm}$ is in great demand for a number of applications, such as ultrahighspeed coherent optical transmission [1,2], optical arbitrary waveform generation [3], astronomical spectrograph calibration [4], and microwave frequency 
transfer via optical fiber networks [5]. Many of these applications require optical frequency stabilization of the $\mathrm{GHz}$ optical pulse source. However, it is difficult to realize such a light source with a conventional optical stabilization scheme using a one-octave method $[6,7]$, because of the low pulse energy. To solve this problem, repetition rate multiplication with a Fabry-Perot (FP) cavity filter was demonstrated [4, 8]. For example, a $25 \mathrm{GHz}$-spaced optical frequency stabilized comb was generated by filtering a $250 \mathrm{MHz}$-spaced comb with two FP cavity filters, whose resonance frequencies were actively locked to the original $250 \mathrm{MHz}$-spaced comb [4]. In this scheme, the laser system becomes complex due to the use of multiple stabilization circuits.

On the other hand, we have demonstrated a new frequency standard signal source called a "Cs optical atomic clock" [9, 10, 11]. The clock source uses a regeneratively mode-locked fiber laser (MLFL) [12] as a microwave oscillator, whose repetition rate and optical frequency are locked to a $9.19 \mathrm{GHz}$ Cs resonance line and a $1.54 \mu \mathrm{m} \mathrm{C}_{2} \mathrm{H}_{2}$ absorption line, respectively [11]. An MLFL can emit a transform-limited picosecond pulse train with low jitter and an optical comb signal with a narrow linewidth of a few $\mathrm{kHz}$ [13]. The Cs optical atomic clock can directly generate an optical frequency stabilized pulse train with a $\mathrm{GHz}$ repetition rate.

In this paper, we demonstrate the improved performance of the optical frequency stabilized Cs optical atomic clock. By adopting a phase modulator instead of an intensity modulator as a mode-locker, we shortened the output pulse width by $50 \%$. The long-term stability of the optical frequency was also greatly improved by precisely controlling the laser cavity temperature.

\section{Configuration of optical frequency stabilized Cs optical atomic clock}

The configuration of our Cs optical atomic clock is shown in Fig. 1, in which the thick lines are optical paths and the thin lines are electrical paths. In this scheme we replace a 5 or $10-\mathrm{MHz}$ quartz oscillator and multipliers with

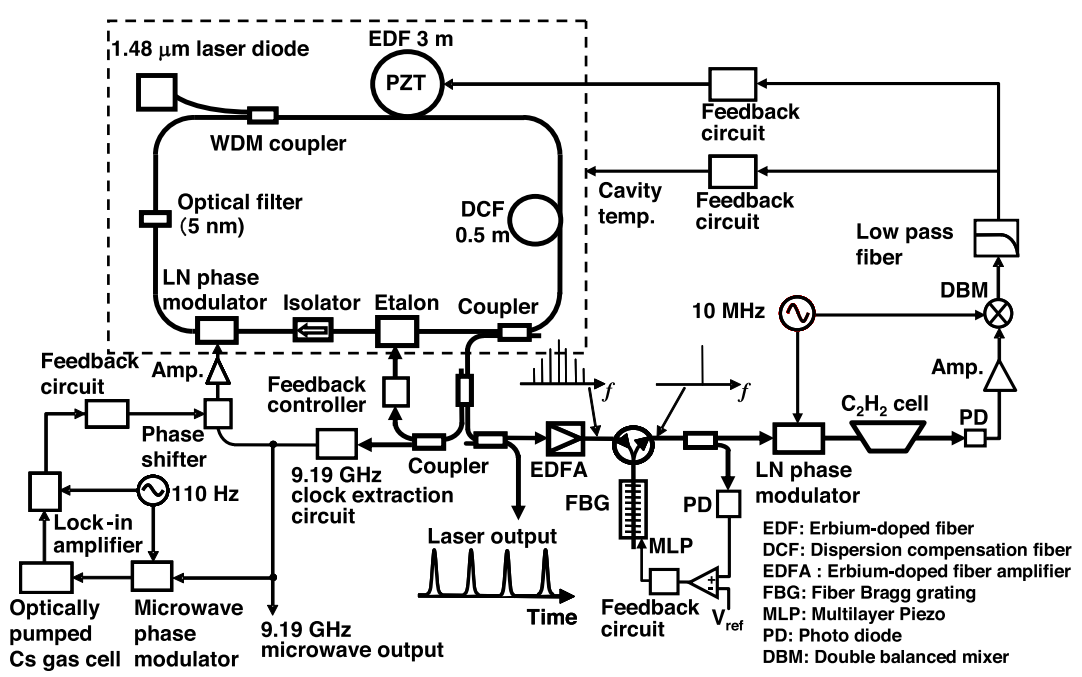


an MLFL that directly emits a microwave signal at 9.19 GHz. The MLFL consists of a $3-\mathrm{m}$ long erbium-doped fiber (EDF), a 0.5-m long dispersion compensation fiber (DCF), a 30\% output coupler, an optical etalon, an isolator, a $\mathrm{LiNbO}_{3}$ phase modulator, an optical bandpass filter, and a WDM coupler. All the fibers in the cavity are polarization-maintaining to prevent polarization fluctuation. The cavity length is $5.9 \mathrm{~m}$, which corresponds to a cavity mode spacing of $34.5 \mathrm{MHz}$. The pumping source consists of a $1.48 \mu \mathrm{m}$ InGaAsP laser diode (LD), and the filter bandwidth is $5 \mathrm{~nm}$. The oscillation wavelength can be changed by tuning the center wavelength of the bandpass filter over $30 \mathrm{~nm}$. Part of the EDF is wound on a PZT to allow us to change the cavity length by applying a voltage signal. We install an optical etalon with a finesse of 115 , and control its peak frequency to realize mode-hop-free laser operation [14]. To obtain a short pulse train from the laser, a phase modulator is employed as a mode-locker instead of the intensity modulator used in our previous work [11], and the DCF is installed to adjust the cavity average dispersion to near zero.

As a microwave frequency standard to stabilize the repetition rate, we adopt an optically pumped Cs gas cell with a stability of $7.5 \times 10^{-13}$ for an averaging time $\tau$ of $1 \mathrm{~s}$ and $7.0 \times 10^{-14}$ for a $\tau$ of $100 \mathrm{~s}$ [15]. This frequency standard consists of a pumping LD $(852.1 \mathrm{~nm})$ and a gas cell where the cavity resonates at $9.19 \mathrm{GHz}$ in the $\mathrm{TE}_{011}$-mode. A double resonance method, which is realized by the interaction between the pump light and the microwave signal from the MLFL in this gas cell [16], is used to detect a Cs resonance center. The repetition rate of the MLFL is stabilized by changing the microwave phase delay in the regenerative feedback loop [17]. By introducing this technique, the repetition rate of the MLFL can be controlled without disturbing the optical frequency. The error signal used for this stabilization is obtained by detecting the frequency deviation from the Cs resonance center with a phase sensitive detection (PSD) circuit and is fed back to a voltage controlled microwave phase shifter in the feedback loop. As a result, we can generate a $9.19 \mathrm{GHz}$ optical pulse train whose repetition rate has the same stability as that of the Cs resonator [10].

By contrast, the optical frequency of the MLFL is tuned by changing both the cavity length with the PZT and the laser cavity temperature. After amplifying the output optical comb from the laser with an erbium-doped fiber amplifier (EDFA), one of the longitudinal modes near the center frequency is extracted by using a fiber Bragg grating (FBG) with a $1.5 \mathrm{GHz}$ bandwidth. The FBG is mounted on a multilayer piezo actuator, which enables us to tune the optical filter frequency. To match the extracted longitudinal mode with the resonance of $\mathrm{C}_{2} \mathrm{H}_{2}$ molecules, an optical frequency component near the $\mathrm{P}(10)$ absorption line $(1538.8 \mathrm{~nm})$ is extracted through the FBG. Here, we stabilized the amplitude of the extracted longitudinal component to avoid the inclusion of a false error signal caused by amplitude fluctuation in the longitudinal mode. This can be achieved by employing a differential control from a reference voltage, where the longitudinal mode is set at the shoulder of the FBG filter characteristics so that the output power becomes constant. 
The frequency deviation from the $\mathrm{C}_{2} \mathrm{H}_{2}$ absorption line is detected with another PSD circuit. Then the error signal is fed back to the PZT for optical frequency stabilization. We also used a laser cavity temperature control to compensate for slow frequency drifts caused by temperature fluctuations.

\section{Output characteristics of Cs optical atomic clock}

The output characteristics of the Cs optical atomic clock against pump power are shown in Fig. 2. Figure 2 (a) shows the relationship between laser output power and pump power, and Fig. 2 (b) shows the pulse width and timebandwidth product against pump power. In Fig. 2 (a), the threshold pump power was $14.5 \mathrm{~mW}$, and an output power of $7.2 \mathrm{~mW}$ was obtained at a max-

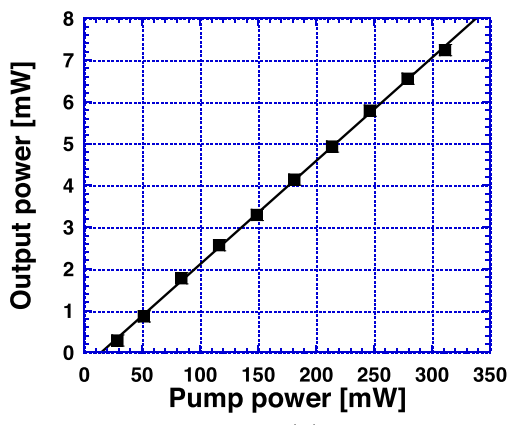

(a)

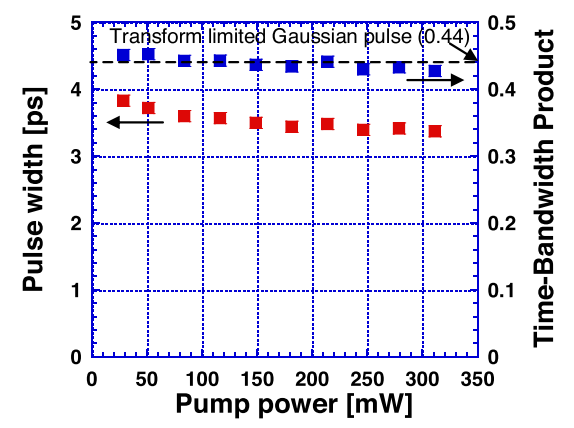

(b)

Fig. 2. Output characteristics of Cs optical atomic clock against pump power: (a) output power, (b) pulse width and time-bandwidth product.

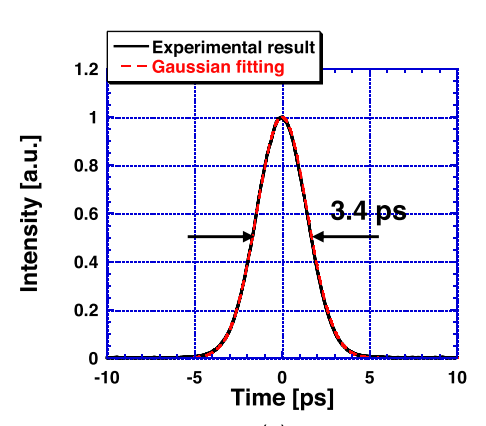

(a)

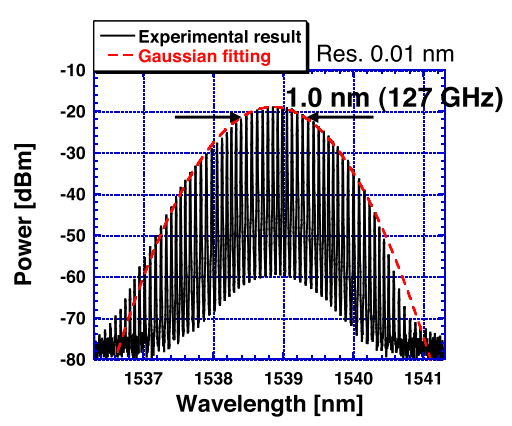

(b)

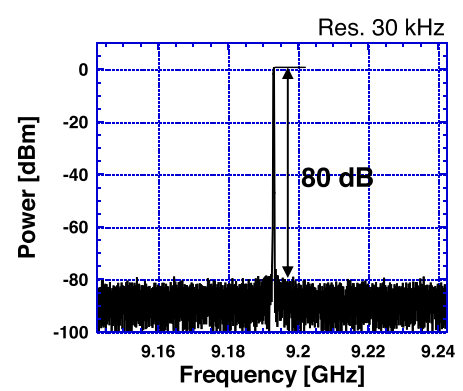

(c)

Fig. 3. Output pulse characteristics of Cs optical atomic clock with a pump power of $310 \mathrm{~mW}$ : (a) autocorrelation waveform, (b) optical spectrum, and (c) $9.19 \mathrm{GHz}$ electrical clock signal. 
imum pump power of $310 \mathrm{~mW}$. Transform limited Gaussian pulse trains with pulse widths of 3.4 to $3.8 \mathrm{ps}$ were obtained over a 15 to $310 \mathrm{~mW}$ pump power range, as shown in Fig. 2 (b). Figure 3 (a) and (b) show an autocorrelation waveform and an optical spectrum with a pump power of $310 \mathrm{~mW}$, respectively. Here, red broken lines are Gaussian fitting curves and these fit well to the experimental results. In Fig. 3 (a), the output pulse width was $3.4 \mathrm{ps}$ after chirp compression using a fiber with an anomalous dispersion of $3.3 \mathrm{ps} / \mathrm{nm}$. In our previous work [11], we used an intensity modulator as a mode-locker and the pulse width was 7.1 ps. By employing a phase modulator instead of the intensity modulator and adjusting the cavity dispersion, the pulse width was shortened by $50 \%$. In Fig. 3 (b), the oscillation wavelength was $1538.8 \mathrm{~nm}$ and the spectral width was $1.0 \mathrm{~nm}(127 \mathrm{GHz})$. Figure $3(\mathrm{c})$ shows the $9.19 \mathrm{GHz}$ clock spectrum detected with an electrical spectrum analyzer. There was only one clock component at $9.19 \mathrm{GHz}$ and the supermode noise was suppressed by more than $80 \mathrm{~dB}$ compared with its peak value.

\section{Improvement in optical frequency stability of Cs optical atomic clock}

A schematic diagram of the temperature control system for the laser cavity is shown in Fig. 4. The laser cavity temperature must be precisely controlled to achieve a good long-term optical frequency stability for the Cs optical atomic clock. To control the temperature, the laser cavity is housed in a double temperature control system where the temperatures of an inner and an outer case are independently controlled. A machined aluminum case with a size of $180 \times 130 \times 20 \mathrm{~mm}^{3}$ is used as the inner case, in which the laser cavity is installed. The inner case temperature is controlled by an error signal detected from a PSD circuit with a $\mathrm{C}_{2} \mathrm{H}_{2}$ molecular gas cell as shown in Fig. 1. On the other hand, the temperature of the outer aluminum case with a size of $200 \times 150 \times 40 \mathrm{~mm}^{3}$ is controlled at a constant temperature by using a commercially available temperature controller.

The measured temperature fluctuations of the outside air (monitor 1) and the inner case (monitor 2) are shown in Fig. 5. Figure 5 (a) and 5 (b)

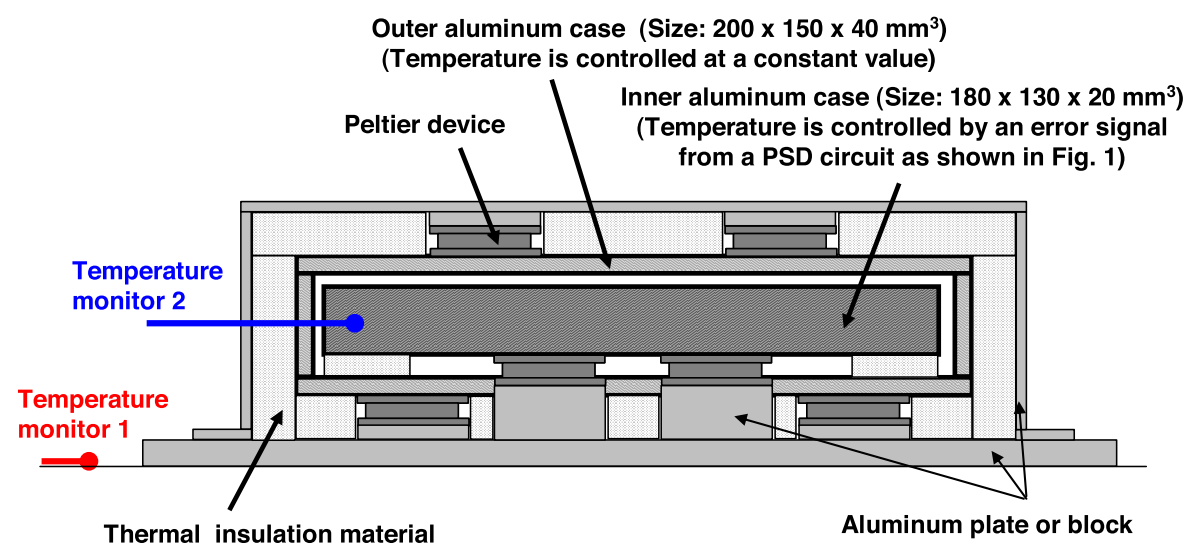

Fig. 4. Configuration of double temperature control system of laser cavity. 


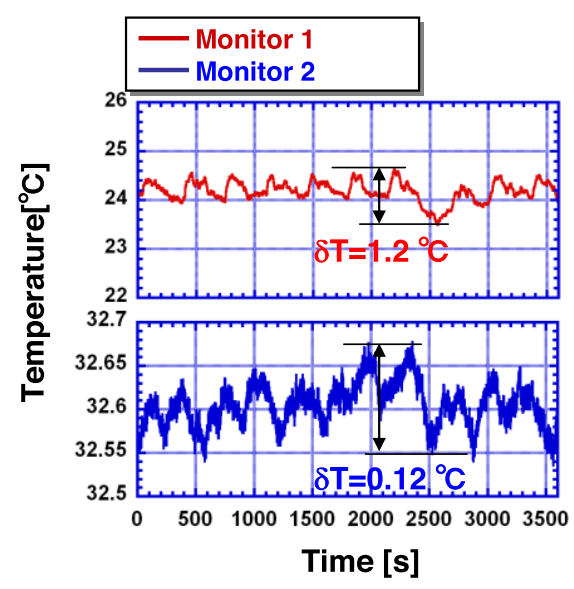

(a)

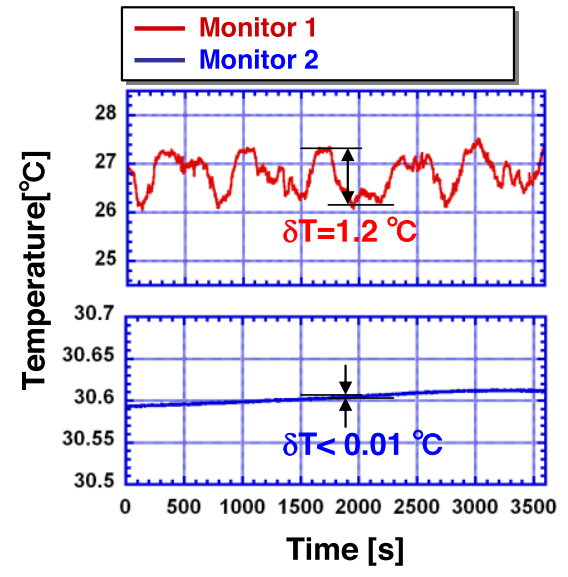

(b)

Fig. 5. Temperature fluctuations of outside air and inner case (a) without and (b) with outer case temperature control.

show the results obtained without and with outer case temperature control, respectively. In Fig. 5 (a), the outside temperature was changed by $1.2^{\circ} \mathrm{C}$ in a period of several hundreds of seconds, resulting in a temperature fluctuation of the inner case of $0.12^{\circ} \mathrm{C}$. On the other hand, by employing the outer case temperature control as shown in Fig. 5 (b), the inner case temperature fluctuation was suppressed to less than $0.01^{\circ} \mathrm{C}$, which was below the measurement resolution. Although there is a long-term temperature drift in Fig. 5 (b), the effect on the optical frequency change can be compensated by the inner case temperature control with the PSD circuit.

We evaluated the optical frequency stability of the Cs optical atomic clock from the beat note signals with a $\mathrm{C}_{2} \mathrm{H}_{2}$ frequency stabilized fiber laser [18]. The measured optical frequency fluctuations are shown in Fig. 6. Figure 6 (a) and 6 (b) are the measurement results obtained without and with the double temperature control system as shown in Fig. 4. In Fig. 6 (a), a frequency fluctuation was observed for a period of several hundred seconds corresponding to the outside temperature fluctuation as shown in Fig. 5 (a). On the

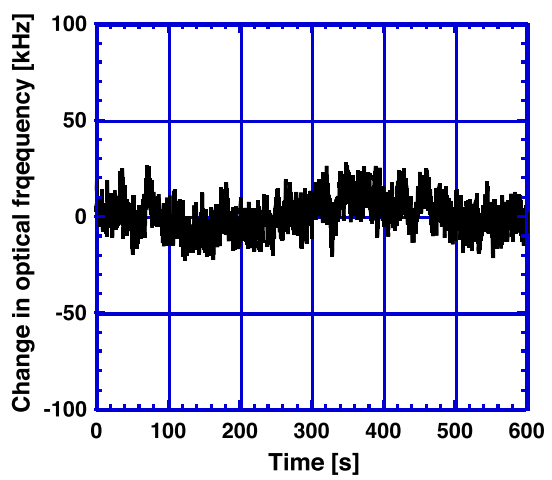

(a)

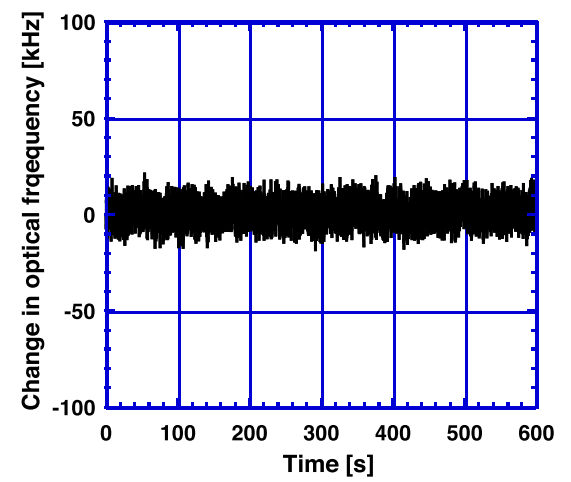

(b) (c) IEICE 2012

DOI: $10.1587 /$ elex.9.1496 Received August 24, 2012 Accepted September 04, 2012 Published September 28, 2012
Fig. 6. Change in the optical frequency of Cs optical atomic clock (a) before and (b) after the improvement of the laser cavity temperature control. 
other hand, the long-term fluctuation was eliminated after the laser cavity temperature control was improved as shown in Fig. 6 (b). Figure 7 shows the square root of the Allan variance estimated from the optical frequency fluctuation shown in Fig. 6 [19]. The black and red plots are results obtained before and after the improvement of the laser cavity temperature control. As seen in Fig. 7, the long-term frequency stability was greatly improved by employing the double temperature control system, resulting in a stability of $6.3 \times 10^{-12}$ for an averaging time of $100 \mathrm{~s}$.

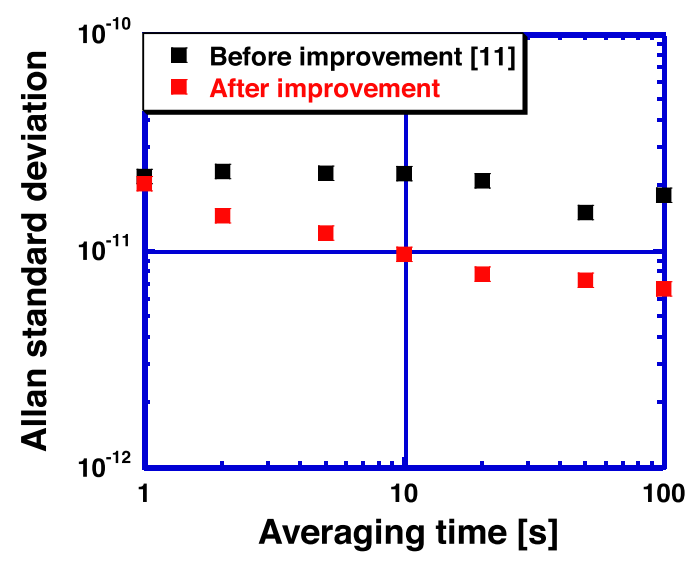

Fig. 7. Optical frequency stability of Cs optical atomic clock.

\section{Conclusion}

We have successfully demonstrated an optical frequency stabilized Cs optical atomic clock with a shortened pulse width and improved long-term optical frequency stability. By using FM mode-locking, the output pulse width was shortened to 3.4 ps. Furthermore, an optical frequency stability for an averaging time of $100 \mathrm{~s}$ was improved from $1.8 \times 10^{-11}$ to $6.3 \times 10^{-12}$ by precisely controlling the temperature of the laser cavity. This Cs optical atomic clock is expected to be useful as an RZ pulse source for ultrahigh-speed coherent transmission as well as for high-precision optical metrology. 